\title{
Identification and reduction of adverse drug reactions
}

This article was published in the following Dove Press journal:

Journal of Healthcare Leadership

6 July 2010

Number of times this article has been viewed

\author{
Dennis F Thompson \\ Randall P Sharp \\ Southwestern Oklahoma \\ State University, Weatherford, \\ Oklahoma, USA
}

Correspondence: Dennis FThompson Southwestern Oklahoma State University, 100 Campus Drive, Weatherford, OK 73096 USA

Tel +I 40560 I 2484

Fax +I 405 60I I20I

Email dennis.thompson@swosu.edu
Abstract: Adverse drug reactions (ADRs) are a significant challenge to health care leaders. Serious ADRs increase the morbidity and mortality of patients and create a significant financial impact on health care costs. There are a number of challenges in identifying and reducing the incidence of ADRs. Worldwide, developed countries primarily utilize voluntary systems to identify ADRs and assess their risk to specific populations of patients. All of these systems have significant limitations. The explosion of biomedical research also challenges the clinician to uncover all the literature available concerning particular ADRs. Once identified, assessing causality in a specific patient-drug scenario can be problematic despite the number of scales, algorithms, and nomograms available. Finally, pharmacogenomics is discussed and the implications of personalized genomic medicine on ADRs are described. Pharmacogenomics promises the best possible hope for a significant reduction of clinically important ADRs in the future, although hurdles remain for its' widespread clinical application.

Keywords: adverse drug reactions, surveillance, pharmacogenomics

\section{Introduction}

Adverse drug reactions (ADRs) constitute a substantial source of morbidity and mortality in health care. Estimates of the costs of ADRs in hospitalized patients range from 2 to 4 billion dollars per year, and in ambulatory patients costs range from 30 to 136 billion dollars per year. ${ }^{1,2}$ The current economic crisis in health care demands close scrutiny of ADRs as a way to reduce overall costs. Of course, only cost-effective methods of identifying and reducing ADRs are likely to be pursued in the future. The purpose of this paper is to review the current literature on ADRs. To accomplish this, we reviewed the English language literature on both identification of ADRs and methods of reducing their frequency.

An ADR is defined by the World Health Organization as any noxious, unintended, and undesired effect of a drug, which occurs at doses used in humans for prophylaxis, diagnosis, or therapy. ${ }^{3}$ Adverse drug reactions are a problem which affects health care systems worldwide. A widely cited meta-analysis of 39 prospective studies in hospitalized patients in the US estimated an overall incidence of serious ADRs of $6.7 \%$. Furthermore, it has been estimated that ADRs cause up to $7 \%$ of all hospital admissions in the UK and $13 \%$ of all admissions to internal medicine clinics in Sweden. ${ }^{4}$ In New Zealand, $12.9 \%$ of all hospital admissions are due to adverse drug events. ${ }^{5}$

A major problem with the identification and reporting of ADRs is the lack of adequate surveillance systems. In the US, the Food and Drug Administration (FDA) relies on voluntary reports to its Adverse Event Reporting System (AERS) from health 
care professionals. ${ }^{6}$ Underreporting of ADRs continues to plague all the current surveillance systems around the world. This is further compounded by the FDA having limited funding, staff, and resources to monitor the AERS. ${ }^{7-9}$ Other countries, such as France, New Zealand, Japan, and the UK also rely on voluntary reports of ADRs. However, important differences exist worldwide in how proactive the surveillance systems are. In the UK, ADRs are actively solicited through the Prescription-Event Monitoring System, which surveys prescribers regarding any adverse experiences among the first 10,000 people who use a drug. Active surveillance in Japan is conducted for the first six months a drug is used, which involves repeated announcements about vigilance and queries to physicians. ${ }^{8}$ In New Zealand, adverse drug reactions are reported to the Center for Adverse Reactions Monitoring, ${ }^{9}$ but reporting of ADRs is not standardized due to differences between district health boards. ${ }^{10}$ These examples of identification and reporting mechanisms from various countries are merely representative of wider practices that occur in other countries around the world.

Regardless of country, the majority of ADRs are reported as individual case reports, which have a number of limitations. Because of the scant number of reports, insufficient causal evidence exists to connect an individual drug with an associated adverse effect. ADRs are divided into Type A and Type B by the Rawlins and Thompson classification scheme, whereby Type A reactions are predictable and commonly related to the pharmacologic actions of the drug (see Table 1). ${ }^{4,11}$ Conversely, Type B reactions are unpredictable and uncommon and usually not related to the pharmacologic actions of the drug. ${ }^{12}$ Case reports are also of limited value because they are often a point-in-time evaluation and not investigated further. Because of the inadequacy of evidence, much of these ADR data are not incorporated into drug reference sources. ${ }^{13}$

Table I Characteristics of Type A and Type B reactions ${ }^{4,11}$

\begin{tabular}{ll}
\hline Type A & Type B \\
\hline $\begin{array}{l}\text { Common, predictable (80\% all } \\
\text { reactions) }\end{array}$ & Uncommon, unpredictable \\
May occur in any individual & $\begin{array}{l}\text { Occurs only in susceptible } \\
\text { individuals }\end{array}$ \\
$\begin{array}{l}\text { Usually related to dose } \\
\text { Usually mild in severity } \\
\text { Related to pharmacologic drug } \\
\text { actions }\end{array}$ & $\begin{array}{l}\text { No soportionally more severe } \\
\text { High morbidity but low mortality } \\
\text { Includes toxicity, overdose, side } \\
\text { effect, secondary effect, and drug } \\
\text { interaction }\end{array}$ \\
\hline
\end{tabular}

The advent of modern computer technology and the promise of pharmacogenomics may help overcome some of these issues. Data mining techniques involve analyzing large volumes of ADR data by computer programs to detect previously unknown or suspected ADRs. As discussed later in this paper, these methods hold the promise of efficiently sifting through huge amounts of ADR reports. ${ }^{14,15}$ However, data mining techniques have both advantages and disadvantages. Pharmacogenomics is likely to help in the future for both preventing and reporting of ADRs. In the future, genetic profiles of patients may help predict who may respond well to a drug and who may experience an ADR. ${ }^{4}$

\section{Literature problem}

The literature problem in pharmacovigilance has both a quantitative and qualitative aspect. The quantitative issue is the sheer mass of biomedical literature currently being produced and the qualitative issue is the difficulty in determining a clear causal relationship between a drug and an adverse drug event.

Recent estimates of the explosion of biomedical literature suggest that there are close to 40,000 biomedical journals that are currently being published worldwide. ${ }^{16-18}$ A clinician today would have to read close to 10,000 articles per day to keep up with all the new information of biomedical importance. Drug therapy review articles have increased by over 10,000\% from 1966 to 2003. Biomedical articles on ADRs in humans now number approximately 10,500 articles/year. ${ }^{16}$ This expansion of information makes identifying all available ADR data on a drug extremely difficult. Even after combining various literature databases, such as Medline, Embase, Science Citation Index, and International Pharmaceutical Abstracts, relevant papers can be missed. This is particularly true for non-English language biomedical literature. Therefore, the ADR literature suffers not from a lack of information but from an overwhelming glut of information.

While the sheer volume of biomedical literature could be viewed as either positive or negative, the quality of the data available on adverse drug events would have to be categorized as lacking. Determining causality in adverse drug events is an inexact science. ${ }^{19-23}$ A number of nomograms, scales, and algorithms have been published to reduce bias and increase reproducibility. However, none of the available methods can accurately and reliably quantify the relationship between a potential adverse event and a drug. ${ }^{22-23}$

Randomized, controlled, clinical trials are usually a clinician's best source of evidence on the efficacy of a drug. Although most clinical trials are scaled to determine small, 
incremental benefits of a drug, clinical trials also provide an excellent potential opportunity to quantify risks. Unfortunately, recent systematic reviews of this subject have found adverse event reporting to be insufficient or misleading in many controlled clinical trials. ${ }^{24-26}$ Pitrou et al ${ }^{25}$ recently re-reviewed 133 randomized clinical trials in six high-impact factor journals. No information on withdrawal of patients due to an adverse event was reported in $47.4 \%$ of these articles. No information on severe adverse events was reported in $27.1 \%$ of the articles. The accompanying editorial to this paper describes adverse event reporting in randomized trials as "neglected, restricted, distorted, and silenced". ${ }^{24}$

Because of the overwhelming amount of data available, data mining techniques have also been applied to adverse drug events. ${ }^{27}$ Data mining involves the use of statistical and probability programs applied to large databases to identify previously unrecognized ADRs or evaluate trends. Using Bayesian algorithms, patterns may be identified from databases containing virtually millions of ADRs. Potential databases can involve national voluntary reporting systems such as the US FDA or the UK Prescription Event Monitoring System. Other databases that can be used include large health insurance programs, electronic medical records, and even poison information centers. Of course, like most epidemiologic data, data mining can only suggest associations between drugs and adverse events and does not prove causality. Furthermore, self-deception bias can occur when a data miner with a strong incentive to believe in a particular outcome may consciously or subconsciously try to avoid results that contradict pre-existing expectations. ${ }^{15}$ However, these data can detect signals of possible drug-adverse event associations and suggest fruitful avenues to employ scarce resources to collect prospective data. Used appropriately, data mining can be a valuable tool in pharmacovigilance. ${ }^{27}$

\section{Systems approach to identifying and reducing adverse drug reactions}

Other techniques utilizing computer technology have been successful at detecting and preventing ADRs as well. Prevention of ADRs has been documented with physician order entry systems whereby a physician inputs the drug order directly into a computer system for subsequent administration/dispensing. ${ }^{28}$ Bates et $\mathrm{al}^{29}$ have shown this method to be an effective way to decrease ADRs. Their study was done at a large tertiary US hospital over a 15-month period. Nonintercepted serious medication errors decreased by $55 \%$ from 10.7 events per 1000 patient days to 4.86 events per 1000 patient days $(P=0.01)$. Another successful method that has been used is a medication reconciliation program, which involved a health care provider (nurse, physician, pharmacist) obtaining a medication history before a patient was discharged. The relevant study was a controlled trial with 322 patients on general medical inpatient units at two US academic hospitals over a two-month period. A $28 \%$ relative risk reduction in unintentional medication discrepancies with potential for harm occurred compared with the group who did not receive pre-discharge medication history and counseling. ${ }^{30}$

Better utilization of pharmacists has also proven successful in the identification and reduction of ADRs as well as overall health care costs. Two studies, one on an inpatient population and the other on an outpatient population, support this. The first study utilized an inpatient population of patients 80 years or older in Sweden over an eight-month period. Patients were randomized to two groups whereby 186 in the control group received standard care without direct involvement of pharmacists, and 182 patients in the intervention group who had pharmacists directly involved in their care. They found a $16 \%$ reduction in return hospitalization and a $47 \%$ reduction in visits to the emergency department, while drug-related readmissions were reduced by $80 \%$ compared with the control group. The total cost per patient in the intervention group was $\$ 230$ lower than for the control group. ${ }^{31}$ The second study analyzed two randomized controlled trials in the US to determine the effect of pharmacist intervention on adverse drug events and medication errors in outpatients with cardiovascular disease. Compared with the control group, the risk of ADRs was 34\% lower in the intervention group where a pharmacist was involved in patient care. ${ }^{32}$ Zolezzi and Parsotam pointed out that about 5.7\% of ADR reports are submitted by pharmacists in New Zealand, compared with about $70 \%$ of ADR reports submitted to the MEDWATCH program in the US. The article further explains how to get pharmacists better involved in ADR reporting in New Zealand. ${ }^{9}$

Finally, better reporting and reduction of ADRs could be accomplished by improvements in surveillance systems and the emergence of pharmacogenomics. Common improvements to the surveillance system, regardless of country, may include better funding, increased staff dedicated to detecting ADRs, less reliance on case reports, improvements in data mining algorithms, and less reliance on purely voluntary ADR reporting. ${ }^{12,33}$ As more genetic information becomes available on how patients metabolize various drugs, pharmacogenomics may both serve as a tool to develop new medications and enhance current drug-surveillance strategies. 
ADR surveillance would be improved by having results of genetic testing stored in a database, where this information could be incorporated into data mining algorithms. ${ }^{4}$ With combinations of the best strategies employed, patient care regarding ADRs would be greatly improved.

\section{Promise of pharmacogenomics}

Type B adverse drug reactions constitute a group of adverse events which are generally unpredictable in nature and not easily related to the known pharmacology of the therapeutic agent. In many cases, these adverse drug reactions are related to genetic variants which cause susceptibility to certain adverse events. The use of genetic information as an aid to improve pharmacotherapeutic outcomes and individualize drug therapy is called pharmacogenomics. ${ }^{34-42}$

The use of pharmacogenomics to reduce adverse drug events can be broken down into two areas. First, there is the effect of genetic factors influencing the pharmacokinetics of a drug and, second, is the effect of genetic variations on the pharmacology and toxicology of a drug. An example of a drug that would fall into the first category is azathioprine. Genetic variation in the enzyme thiopurine-S-methyltransferase is known to increase the risk of myelotoxicity. Toxic epidermal necrolysis caused by carbamazepine is an example of the second mechanism. Identification of patients with variants in the HLA-B1502 alleles can reduce the incidence of this serious dermatologic adverse event. ${ }^{35}$ The US FDA recommends relevant genetic testing of patients prescribed azathioprine and carbamazepine to reduce serious drug toxicities. Recently, new information on the pharmacogenomic variations with warfarin ${ }^{42}$ and $\operatorname{statin}^{41}$ drugs has added to the growing list of therapeutic agents where genetic testing may reduce common adverse events. In fact, the number of drugs approved by the FDA with pharmacogenomic information as part of their drug labeling has increased dramatically in the past 60 years (Figure 1). ${ }^{34}$ Despite this surge in new pharmacogenomic information, the clinical application of this data has been minuscule. ${ }^{37,39}$ The promise of making a significant impact on adverse drug events appears to be in the future.

Phillips et $\mathrm{al}^{36}$ recently conducted a systematic literature review of the potential role of pharmacogenomics in reducing adverse drug events. They evaluated 18 adverse drug reaction studies with detailed inclusion criteria and 22 articles reviewing variant alleles of drug metabolizing enzymes. Using predefined criteria and standardized abstracting forms, these authors identified 27 drugs that were frequently cited in the ADR literature. Of these 27 drugs, 59\% are metabolized by at least one enzyme known to possess a variant allele compared with a randomly selected group of drugs of which only $7 \%-22 \%$ is metabolized by a variant allele $(P<0.001)$. These data provide an early evidenced-based foundation for the reduction in clinically important adverse drug events by pharmacogenomics.

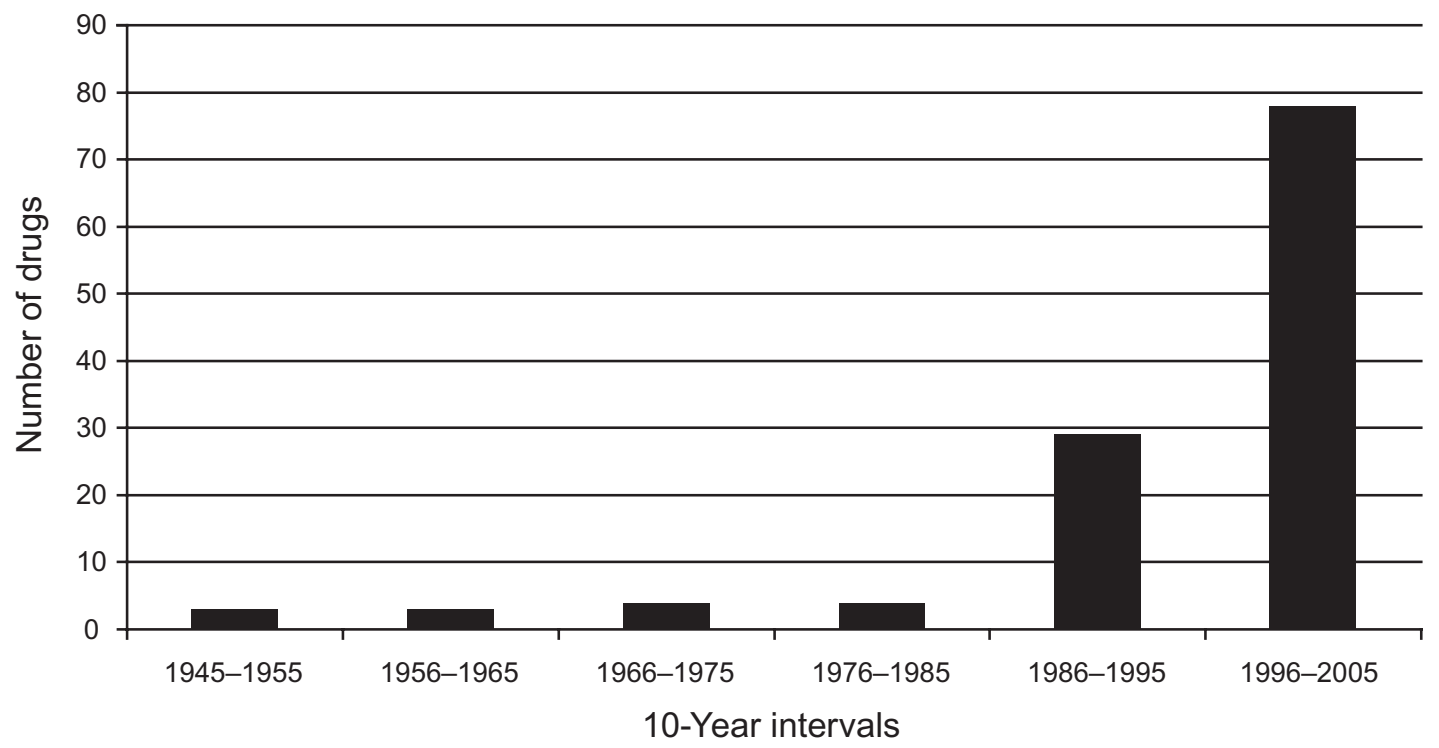

Figure I Number of drugs that were approved with pharmacogenomic information in their drug labels during each 10-year period from 1945-2005. During the 60 years covered by this analysis, 121 drugs were approved that have biomarker information in current product labeling. Reproduced with permission from Frueh FW, Amur S, Mummaneni P, et al. Pharmacogenomic biomarker information in drug labels approved by the United States Food and Drug Administration: Prevalence of related drug use. Pharmacotherapy. 2008;28:992-998..$^{34}$ Copyright (C) 2008 American College of Clinical Pharmacy. 


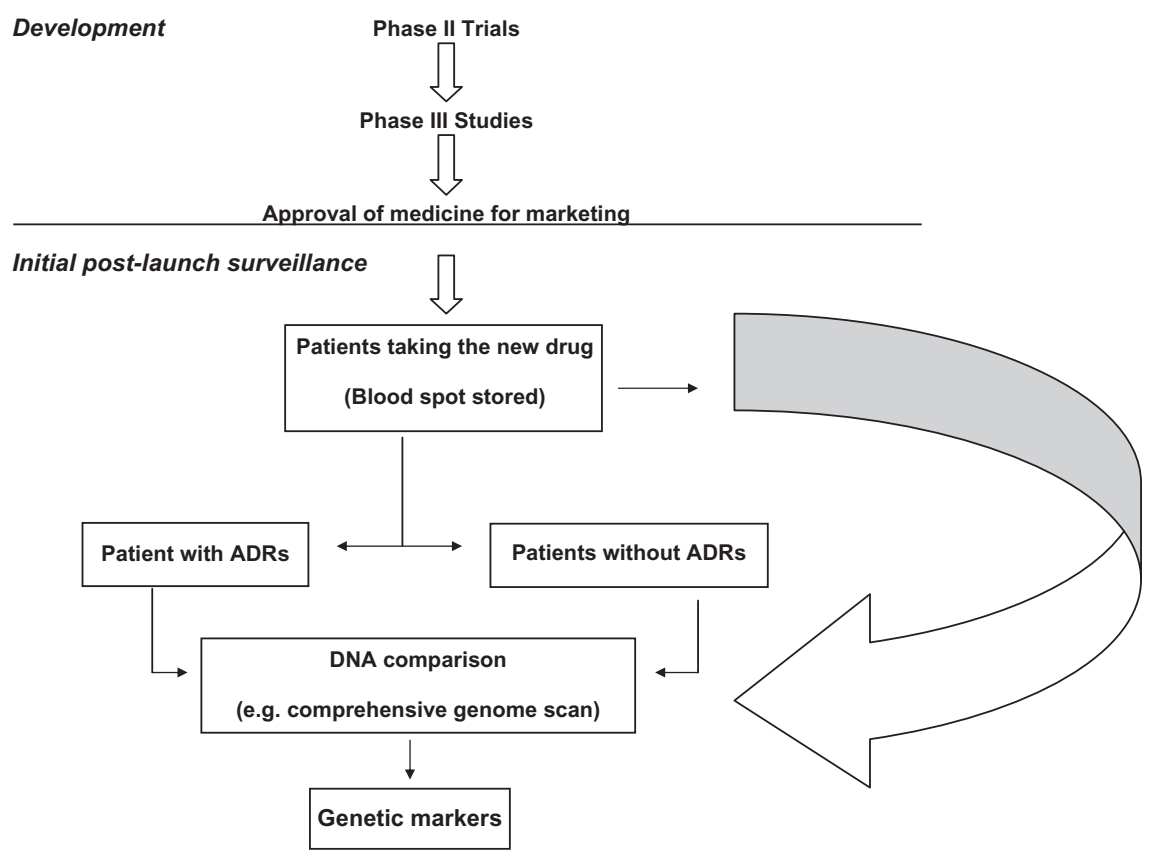

Figure 2 Using pharmacogenomics as part of the drug discovery and surveillance process. Reproduced with permission from Severino G, Del Zompo M. Adverse drug reactions: Role of pharmacogenomics. Pharm Res. 2004;49(4):363-373. ${ }^{4}$ Copyright @ 2004 Springer.

Serverino and Del Zompo ${ }^{4}$ have proposed a model (Figure 2) to collect pharmacogenomic data as part of the post-approval surveillance process. As new drugs are approved for use in the general population, patients who experience rare or severe ADRs should have comprehensive genetic scans which would be compared with those who do not experience such reactions. This approach would allow genetic markers to be identified for patients at risk for rare or severe ADRs. This would be a major advance over the voluntary reporting systems and limited post-marketing clinical trial data. Once established, this genetic bank of information could be used prospectively to identify patients at high risk of severe ADRs.

Recently, there has been a movement in pharmacogenomics away from the DNA-centric focus on single genes. ${ }^{43-44}$ Adverse drug responses, in some cases, are more likely multigenetic traits with numerous genes involved in predisposing patients to ADRs. Transcriptomics, proteomics, metabonomics, and epigenomics are postgenomic technologies that will likely be the next avenues for new knowledge and eventual application in clinical medicine. The huge volume of information created by these genomic and postgenomic disciplines has created the new field of informatics, which endeavors to categorize, store, and retrieve critical information for specific applications. ${ }^{43-48}$ Unfortunately, a detailed discussion of these important new scientific fields is outside the scope of the present paper.
While pharmacogenomics may provide novel solutions to the problem of ADRs, there are significant questions that will need to be addressed before widespread application occurs. At present, there is little application in clinical medicine of pharmacogenetic information to reducing ADRs. Second, there may be increasing difficulties differentiating pharmacogenomic influences versus nongenetic influences, such as age, sex, nutrition, weight, and organ function. Third, whatever screening or mechanistic tools emerge from pharmacogenetic research, they must be cost-effective to gain broad acceptance. Fourth, ADRs are likely to be multigenetic phenomena which may prove more difficult to characterize than first thought. ${ }^{43-44}$ Finally, environmental and other epigenetic influences may play a larger role in the future as this discipline develops. ${ }^{44}$

\section{Disclosure}

The authors disclose no conflicts of interest.

\section{References}

1. Bates DW, Spell N, Cullen DJ, et al. The costs of adverse drug events in hospitalized patients. JAMA. 1997;277(4):307-311.

2. Johnson JA, Bootman LJ. Drug-related morbidity and mortality: A cost-of-illness model. Arch Intern Med. 1995;155(18):1949-1956.

3. Ross SD. Drug-related adverse events: A readers' guide to assessing literature reviews and meta-analyses. Arch Intern Med. 2001;161(8):1041-1046.

4. Severino G, Del Zompo M. Adverse drug reactions: Role of pharmacogenomics. Pharm Res. 2004;49(4):363-373.

5. Chamberlin N. The folly of rewarding silence while hoping for open reporting of adverse medical events - how to realign the rewards. $N Z$ Med J. 2008;121(1282):58-66. 
6. McClellan M. Drug safety reform at the FDA - pendulum swing or systematic improvement. $N$ Engl J Med. 2007;356(17):1700-1702.

7. Woosley RL. Discovering adverse reactions: Why does it take so long? Clin Pharmacol Ther. 2004;76(4):287-289.

8. Trontell A. Expecting the unexpected - drug safety, pharmacovigilance, and the prepared mind. N Engl J Med. 2004;351(14):1385-1387.

9. Zolezzi M, Parsotam N. Adverse drug reaction reporting in New Zealand: Implications for pharmacists. Ther Clin Risk Manag. 2005;1(3):181-188.

10. Kaufman DW, Shapiro S. Epidemiological assessment of drug-induced disease. Lancet. 2000;356:1339-1343.

11. Gruchella RS. Clinical assessment of drug-induced disease. Lancet. 2000;356:1505-1511.

12. Loke YK, Price D, Derry S, Aronson JK. Case reports of suspected adverse drug reactions - systematic literature survey of follow-up. $\mathrm{Br}$ Med J. 2006;332(7537):335-339.

13. Hauben M, Aronson JK. Defining 'signal' and its subtypes in pharmacovigilance based on a systematic review of previous definitions. Drug Saf. 2009;32(2):99-110.

14. Szarfman A, Tonning JM, Doraiswamy PM. Pharmacovigilance in the 21st century: New systematic tools for an old problem. Pharmacotherapy. 2004;24(9):1099-1104.

15. Hauben M, Patadia V, Gerrits C, Walsh L, Reich L. Data mining in pharmacovigilance. Drug Saf. 2005;28(10):835-842.

16. Thompson DF, Williams NT. Tracking the growth of drug therapy literature using PubMed. Drug Inf J. 2007;41:449-455.

17. Wyatt J. Use and sources of medical knowledge. Lancet. 1991;338: 1368-1378.

18. Kelly K. Health sciences library: Future trends. The Irish Library. 2009;18(1):12-17.

19. Karch FE, Lasagna $L$. Toward the operational identification of adverse drug reactions. Clin Pharmacol Ther. 1977;21:247-254.

20. Kramer MS, Leventhal JM, Hutchinson TA, Feinstein AR. An algorithm for the operational assessment of adverse drug reactions. JAMA. 1979;242:623-632.

21. Naranjo CA, Busto U, Sellers EM, et al. A method for estimating the probability of adverse drug reactions. Clin Pharmacol Ther. 1981;30:239-245.

22. Macedo AF, Marques FB, Ribeiro CF, Teixeira F. Causality assessment of adverse drug reactions: Comparison of the results obtained from published decisional algorithms and from the evaluation of an expert panel, according to different levels of imputability. J Clin Pharm Therap. 2003;28:137-143.

23. Macedo AF, Marques FB, Ribeiro CF. Can decisional algorithms replace global introspection in the individual causality assessment of spontaneously reported ADRs? Drug Saf. 1006;29:697-702.

24. Ioannidis JPA. Adverse events in randomized trials. Arch Intern Med. 2009;169(19):1737-1739.

25. Pitrou I, Boutron I, Ahmad N, Ravaud P. Reporting of safety results in published reports of randomized controlled trials. Arch Intern Med. 2009;169(19):1756-1761.

26. Ioannidis JP, Lau J. Completences of safety reporting in randomized trials: An evaluation of 7 medical areas. JAMA. 2001;285: 437-443.
27. Wilson AM, Thabane L, Holbrook A. Application of data mining techniques in pharmacovigilance. Brit J Clin Pharmacol. 2003;57(2): 127-134.

28. Nilsen EV, Fotis MA. Developing a model to determine the effects of adverse drug events in hospital patients. Am J Health Syst Pharm. 2007;64:521-525.

29. Bates DW, Leape LL, Cullen DJ, et al. Effect of computerized physician order entry and a team intervention on prevention of serious medication errors. JAMA. 1998;280(15):1311-1316.

30. Schnipper JL, Hamann C, Ndumele CD, et al. Effect of an electronic medication reconciliation application and process redesign on potential adverse drug events. Arch Intern Med. 2009;169(8):771-780.

31. Gillespie U, Alassaad A, Henrohn D, et al. A comprehensive pharmacist intervention to reduce morbidity in patients 80 years or older. Arch Intern Med. 2009;169(9):894-900.

32. Murray MD, Ritchey ME, Wu J, Tu W. Effect of a pharmacist on adverse drug events and medication errors in outpatients with cardiovascular disease. Arch Intern Med. 2009;169(8):747-763.

33. Curfman GD, Morrissey S, Drazen JM. Blueprint for a stronger food and drug administration. $N$ Engl J Med. 2006;355(17):1821.

34. Frueh FW, Amur S, Mummaneni P, et al. Pharmacogenomic biomarker information in drug labels approved by the United States Food and Drug Administration: Prevalence of related drug use. Pharmacotherapy. 2008;28:992-998.

35. Nakamura Y. Pharmacogenomics and drug toxicity. $N$ Engl J Med. 2008;359(8):856-858.

36. Phillips KA, Veenstra DL, Oren E, Lee JK, Sadee W. Potential role of pharmacogenomics in reducing adverse drug reactions. A systematic review. JAMA. 2001;286:2270-2279.

37. Evans WE, Relling MV. Pharmacogenomics: Translating functional genomics into national therapeutics. Science. 1999;286:487-491.

38. Howland RH. Pharmacogenetics and pharmacovigilance. Drug Saf. 2009;32(3):265-270.

39. Evans WE, McLeod HL. Pharmacogenomics - drug disposition, drug targets, and side effects. N Engl J Med. 2003;348(6):538-549.

40. Welke RA, Lin DW, Rodev DM, et al. Identifying genetic risk factors for serious adverse drug reactions: Current progress and challenges. Nature. 2007;6:904-916.

41. The SEARCH collaborative group. SLCO1B1 variants and statininduced myopathy - A genome-wide study. N Engl J Med. 2008;359(8): 789-799.

42. The International Warfarin Pharmacogenetics Consortium. Estimation of the warfarin dose with clinical and pharmacogenetic data. $N$ Engl $J$ Med. 2009;360(8):753-764.

43. Barone C, Mousa SS, Mousa SA. Pharmacogenomics in cardiovascular disorders: Steps in approaching personalized medicine in cardiovascular medicine. Curr Pharmacogenomics Person Med. 2009;2:59-67.

44. Zhang W, Huang RS, Dolan ME. Integrating epigenomics into pharmacogenomic studies. Curr Pharmacogenomics Person Med. 2008;1:7-14.

45. Kiechele FL, Holland-Staley CA. Genomics, transcriptomics, proteomics, and numbers. Arch Pathol Lab Med. 2003;127:1089-1097.

46. Gomase VS, Tagore S. Transcriptomics. Curr Drug Metab. 2008;9(3): 245-249.

Journal of Healthcare Leadership

\section{Publish your work in this journal}

The Journal of Healthcare Leadership is an international, peer-reviewed, open access journal focusing on leadership for the health profession. The journal is committed to the rapid publication of research focusing on but not limited to: Healthcare policy and law; Theoretical and practical aspects healthcare delivery; Interactions between healthcare and society and evidence-based practices;

\section{Dovepress}

Interdisciplinary decision-making; Philosophical and ethical issues; Hazard management; Research and opinion for health leadership; Leadership assessment. The manuscript management system is completely online and includes a very quick and fair peer-review system. Visit http://www.dovepress.com/ testimonials.php to read real quotes from published authors. 